\title{
Model-aided hybrid metrology for surface roughness measurement fusing AFM and SEM data
}

Vassilios Constantoudis, George Boulousis, Kosmas Ellinas and Evangelos Gogolides Institute of Nanoscience and Nanotechnology, NCSR Demokritos, Aghia Paraskevi, Greece

\begin{abstract}
In this paper, we propose a hybrid metrology approach to the measurement and characterization of the nanoroughness of freeform film surfaces. The basic idea is to combine measurement data from Atomic Force and Scanning Electron Microscopes (AFM and SEM respectively) in a synergetic manner exploiting the advantages of both methods and reducing the effects of their shortcomings. The fusion of data is realized through a model reconstruction of the measured rough surface. In particular, the hybrid approach is implemented by obtaining the height distribution function from AFM topographies (given the high accuracy in AFM height measurements) and the autocorrelation function or Fourier transform of the surface morphology from SEM images (given the high spatial resolution in SEM images). These functions are then used as input to an algorithm to generate rough surfaces in which AFM tip size effects are minimized and hence are more accurate statistical representation of the real surface. The output morphologies may be employed to estimate all roughness parameters and metrics and especially the so-called hybrid parameters (active surface area, distributions of surface derivatives and curvatures etc.) which depend on both vertical and spatial roughness aspects. The latter parameters may be critical in many applications where surface roughness is used to control wetting behaviour, light scattering, bioadhesion or wear properties. As an example, we apply the hybrid approach to the estimation of the active surface area of a sample of cyclic olefin film etched in oxygen plasma for wetting control.
\end{abstract}

\section{Introduction}

In nanotechnology, chemistry and/or roughness engineering of film surfaces is a key technique to endow it with new properties and enable novel applications. Concerning surface roughness, typical examples include its critical role in wetting, optical, wear and biomedical behaviour [1-4]. In order to advance the control of surface roughness effects in these areas, we urgently need techniques and methods to provide complete and accurate measurement and characterization of surface morphology.

The most popular techniques for the inspection of surface morphology at nanoscale are the Atomic Force Microscopy (AFM) and the Scanning Electron Microscopy (SEM). Both methods are characterized by outstanding advantages but also suffer from strong limitations.

AFM measurements have very high vertical resolution, $(\sim 0.1 \mathrm{~nm})$ limited only by the electronic and thermal noise of the instrument and at the same time they provide a welldefined representation of surface topography on a square grid of points. However, the in- 
plane or lateral resolution is strongly affected by the tip shape and size leading to broadening of peaks and shrinking of holes.

As shown schematically in Fig. 1, the measured width of a surface protrusion in an AFM image is largely affected by the tip size and shape. The broadening of a protrusion width depends on its relation with tip radius. Various models have been proposed nicely reviewed in the recent paper by A. Yacoot and Koenders [5]. Based on simulations for columnar thin films, it has been argued that to get reliable AFM imaging of real surfaces the surface features should be sufficiently larger than those of the tip radius and have slopes smaller than those of the tip. For example, sharp edges or structures with high aspect ratio will be distorted in AFM images as illustrated in [6]

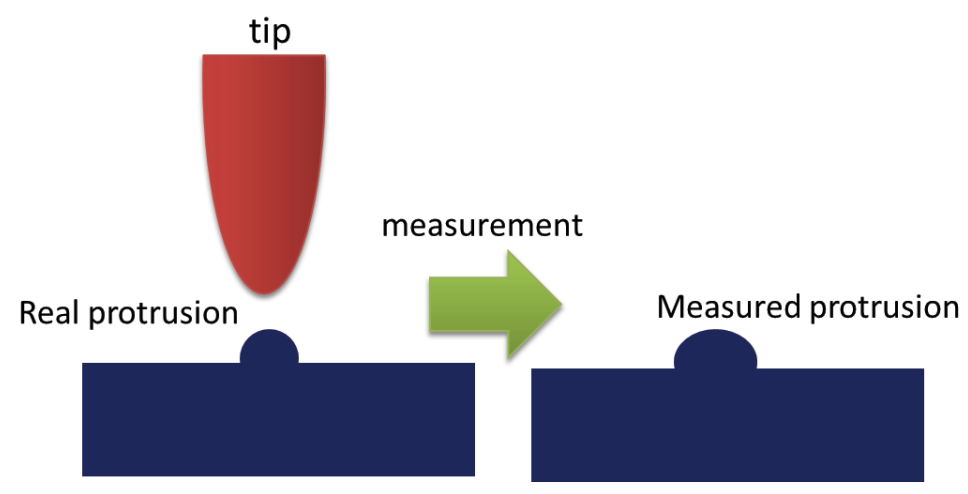

Figure 1. The broadening effect of AFM tip size on the measured diameter of a spherical protrusion.

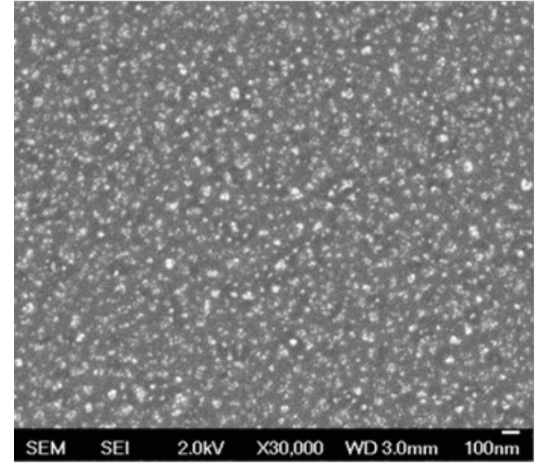

(a)

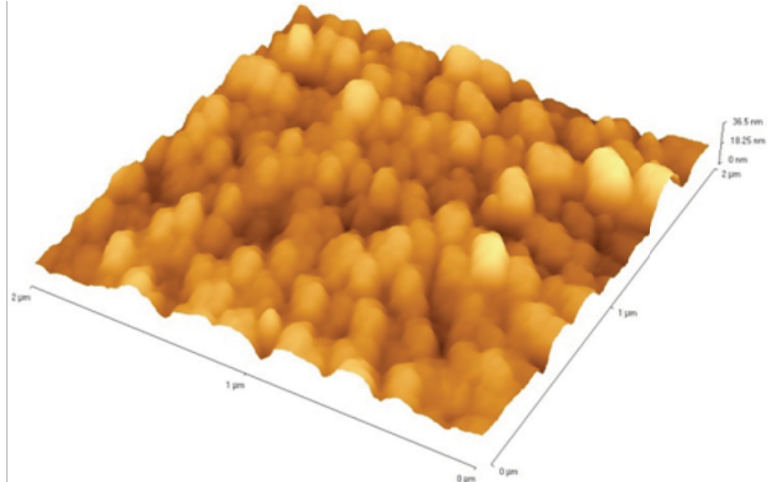

(b)

Figure 2. Typical top-down SEM image (a) and AFM surface (b) with similar measurement ranges of a cyclic olefin film surface after 1 min oxygen plasma etching (for more details see Section 3). Notice the enhanced width of surface protrusions in AFM image coming from tip size and shape effects.

The distorted effects of AFM tip size in real measurements have also been demonstrated by comparison with SEM images. Grutter et al [7] showed that AFM images present artefacts of the order of 20-600 nm with respect to SEM dimensions. In Fig. 2, the same surface of a polymer cyclic olefin film is shown measured by SEM (a) and AFM (b) using almost the same magnification. It is clear that the surface protrusions are displayed thicker in AFM image due to AFM tip convolution. 
To dissolve the issue of detrimental tip effects, ex-situ and in-situ techniques for the determination of tip shape have been proposed in synergy with the definition of genuine surface topographies to reconstruct by erosion the real topography. Moreover, even when no tip shape and surface topography are known by prior measurement, the so called blind method has been proposed to reconstruct tip shape and then by deconvolution the actual surface [8-9]. Despite its recent advances, this method is quite complicated and has noise and resolution implications limiting its applicability [10].

In SEM measurements, the surface is scanned by a narrow electron beam instead of tip and the recorded signal is determined by the secondary electrons emitted from the sample surface. The spatial resolution of SEM is high (usually $<1 \mathrm{~nm}$ ) and thus provides reliable and detailed imaging of the spatial morphology of surface structures. Nevertheless, SEM does not provide direct measurement of surface heights and topography. This drawback has been tried to be overcome through the application of photogrammetry or shape-from-shading techniques to SEM images with limited success though due to their complexity and scale restrictions [1112].

Consequently, both microscopy techniques (AFM and SEM) show advantages and disadvantages in measuring surface roughness. A similar situation has been encountered in other areas of dimensional nano-metrology and especially in the CD metrology of semiconductor structures, where recently the idea of holistic or hybrid metrology has been proposed and elaborated [13-14]. According to it, the results of two or more measuring methods are combined in a synergetic manner to exploit the merits of each method and minimize the impact of their limitations. Up to now, this idea has been implemented to measure the dimensions of lithographic line/space and hole patterns used in the fabrication of integrated circuits where the demand for measurement accuracy is extremely high [15-16].

In this paper, we explore the potential of hybrid / holistic approach to the characterization of the nanoroughness of film surfaces through the combination of data from AFM and SEM measurements. More specifically, from the measurements of AFM we get the height distribution function while from SEM images the autocorrelation function and / or the power spectrum of surface morphology. By a methodological point of view, the innovative aspect of the proposed approach is the implementation of the hybrid concept to the measurement and characterization of a stochastic phenomenon such as the roughness of a surface.

A detailed presentation of our model-aided hybrid approach can be found in Section 2, while Section 3 describes the experimental conditions for the fabrication of the polymer surfaces we are applying the hybrid approach. Then in Section 4, the first results from the application of the proposed hybrid method to the polymeric surfaces are reported and discussed. The drawn conclusions and prospects for future work are summarized in the last Section 5.

\section{Methodology}

The flow diagram of the proposed model-aided hybrid method for the characterization of surface roughness is shown in Fig. 3. The first step is the acquisition of AFM and top-down SEM measurements of the experimental surface. Then, from the AFM images we extract the height distribution function (HDF) of surface points and from the SEM pictures we calculate the autocorrelation function (ACF) of the pixel intensities. The first quantifies the vertical (or 
amplitude) aspect of surface roughness and at first approach its estimation from AFM data is sufficiently accurate given the high AFM accuracy in surface height measurements. The second is a metric of the spatial organization of surface morphology (spatial or horizontal or lateral aspects) and the calculation from top-down SEM images guarantees its accuracy and reliability. The spatial roughness can also be described with other correlation functions (such as height difference correlation function) or transformed in the domain of spatial frequencies with the corresponding power density spectrum estimated by the two-dimensional Fourier transform of the surface.

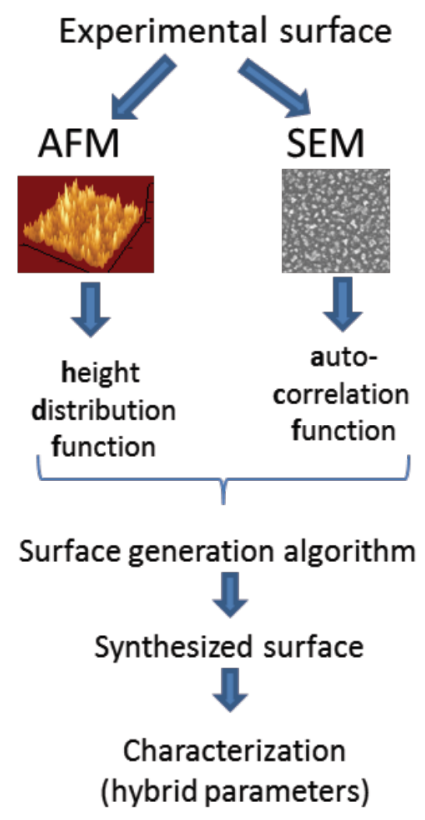

Figure 3. Schematic flow diagram of the hybrid synthesis process AFM measurements and SEM through height distribution function and the autocorrelation function, respectively. The two functions are introduced as input data into surface reconstruction algorithm and deduced surfaces simulating the original test used to characterize the roughness.

After the determination of surface HDF and ACF from the experimental data, we proceed to the generation of rough surfaces based on these functions.

According to roughness theory, a rough surface can be decomposed to its vertical and spatial aspects characterized by the HDF and ACF respectively. Furthermore, using these functions, we can reconstruct in a statistical sense the initial rough surface. The reconstruction of synthetic surfaces was initially implemented with the help of Moving Average or AutoRegressive Moving Average models (MA and ARMA). These methods first create a surface with random heights which are characterized by the desired HDF (typically Gaussian). Then, correlations between the surface points are imposed with parameters determined by the predefined autocorrelation function (usually Gaussian or exponential). Soon it was realized that a variation of the method using the inverse Fourier transformation is most efficient and fast [17-18]. The steps of this method to generate a surface on a grid with $\mathrm{NxN}$ points are the following:

a) With Monte Carlo techniques (acceptance-rejection methods), we generate random numbers arranged on the $\mathrm{NxN}$ surface grid characterized by the HDF derived from the AFM measurements.

b) We calculate the Fourier transform of the random surface generated in a). 
c) We calculate the Fourier transform of the ACF obtained from the analysis of the SEM images.

d) We multiply the amplitudes of the two Fourier transforms and

e) We compute the inverse Fourier transform of the product of d) and with appropriate normalization we get the desired synthetic surface which is characterized by the roughness of the original experimental surface.

The generated surface does not have space and resolution limitations and can be used for statistically reliable measurement of roughness parameters and metrics. The main advantage of this method is that the generated surface can be used for the calculation of the hybrid roughness parameters (actual surface, mean square slope, contact index curve etc.) which cannot be estimated with accuracy from AFM or SEM measurements alone since they depend on both vertical and spatial aspects of surface roughness [ref to ISO or book by Leach].

\section{Application of the method - Experimental data and measurements}

We have applied the proposed hybrid method to the measurement of the surface roughness of a cyclic olefin polymer film etched for $1 \mathrm{~min}$ with oxygen plasma in an RIE reactor. The measurement of the surface morphology has been made by SEM (JEOL JSM -7401f FESEM) and AFM (Veeco CP-II). The energy of the SEM electron beam was $2 \mathrm{keV}$ while the magnification of the analyzed images was $\mathrm{x} 30000$. The measurement by AFM was performed on a square grid of $256 \times 256$ points with a total side length of 2 microns while the scan speed was 2 microns / sec. The width of the tip was estimated $\sim 20 \mathrm{~nm}$. However, the sticking of polymer fragments on it during measurement may make the effective tip radius much larger. In Fig. 2, we show a representative SEM image and AFM surface acquired with the above conditions. Such images have been used in the implementation of the hybrid method presented in the previous paragraph.

Qualitative comparison of two images reveals the widening of surface protrusions in AFM image caused by the effect of tip size and shape. This is manifested quantitatively in the ACF 
of AFM surface which differs significantly from that of the SEM image as shown in Fig. 4.

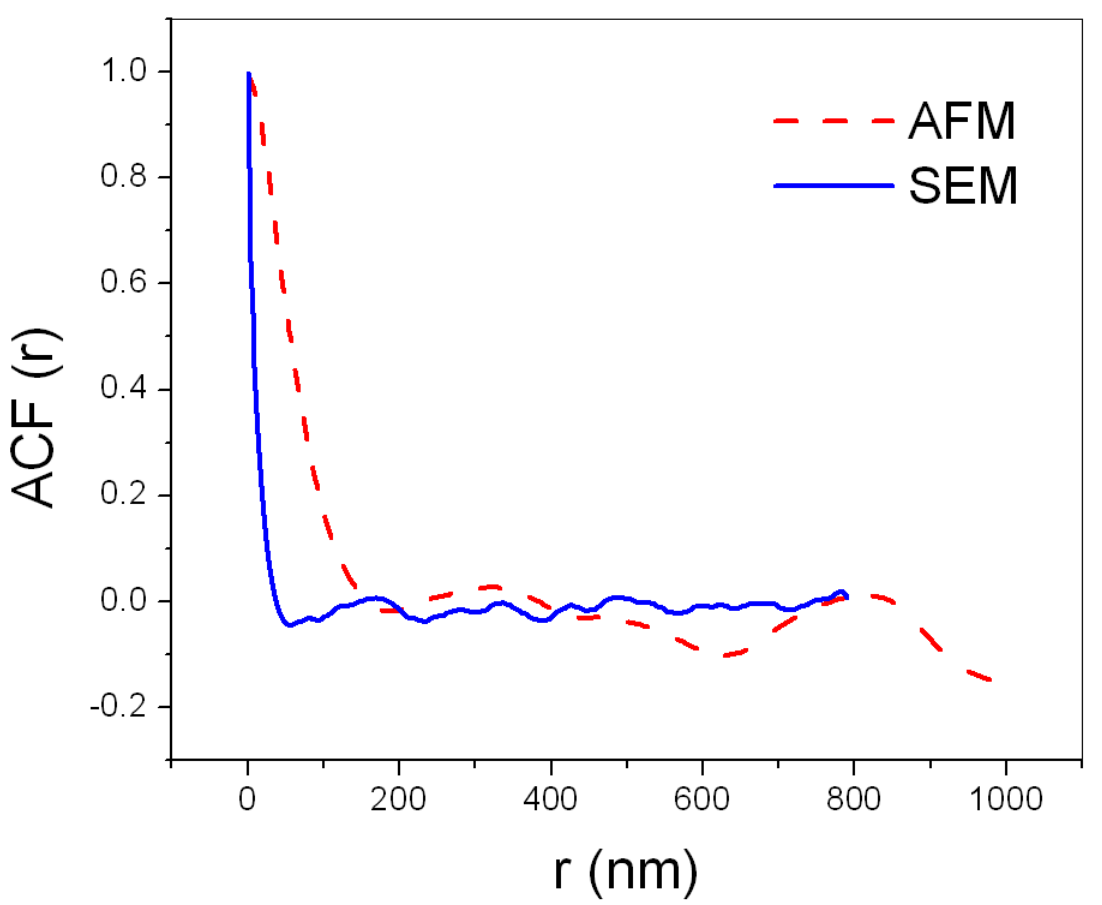

Figure 4. The autocorrelation function ACF (r) of the polymeric surface calculated by the SEM and AFM images (solid and dashed lines respectively). One can clearly notice the more enhanced correlations in AFM surfaces coming mainly from the tip size effects.

\section{Application of the method - Results}

As presented in Section 2, after deriving the microscope images, the next step is the calculation of HDF from the AFM surface and of the 2D ACF of SEM image. For the cyclic olefin film etched by oxygen plasma (see Fig. 2), these quantities are shown in Fig. 5. With input these functions and using Monte Carlo techniques and the inverse Fourier transform, the algorithm generates synthesized rough surfaces such as those shown in Fig. 6. These synthetic surfaces are closer to the real film surfaces than the original AFM or SEM images since they are characterized by the best features of these measurements, i.e. the HDF of AFM measurements and the ACF of SEM images.

Accordingly, these surfaces as long as those generated by a similar input data and methodology can be used to measure more accurately the surface roughness parameters and functions. This is crucial for the hybrid parameters which depend both on the vertical (amplitude) and spatial (lateral) aspects of roughness. For example, calculated the ratio of the actual surface area of the nominal and found $1.35 \pm 0.03$ much higher than the value of 1.1 estimated by AFM surface analysis. The uncertainty in the above estimation is actually yhe standard deviation calculated over many generated surfaces. 

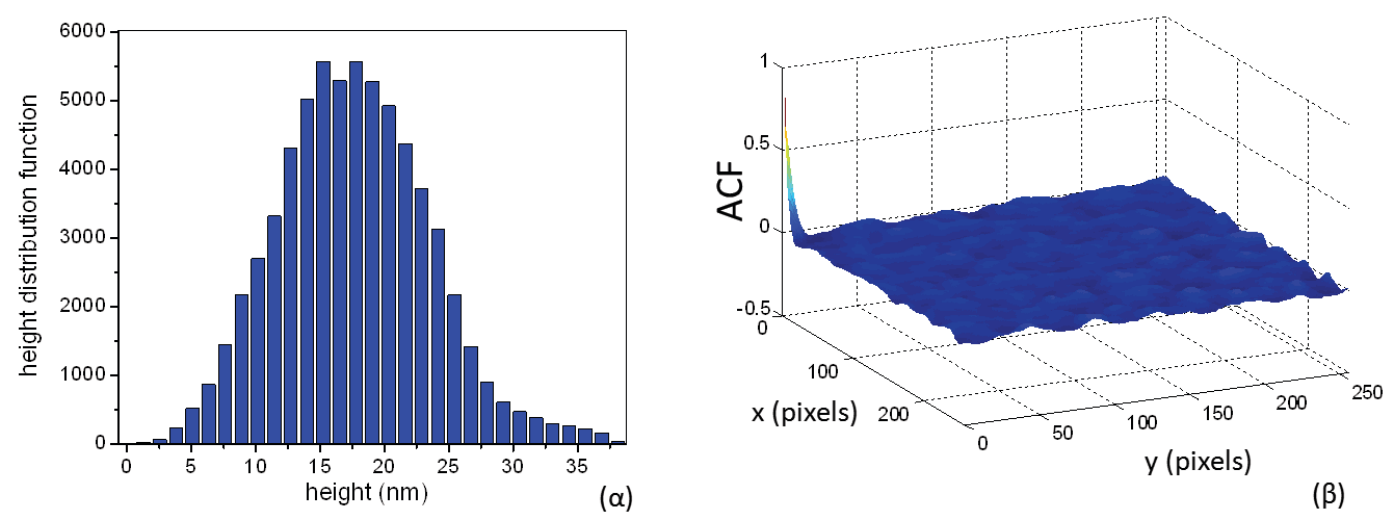

$(\alpha)$

( $\beta)$

Figure 5. Distribution function of the surface heights measured by AFM (a) and 2D autocorrelation function ACF (r) as calculated by the SEM image (b)

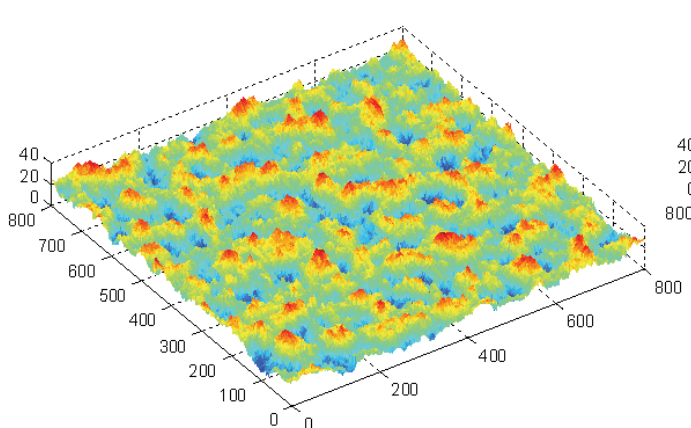

$(\alpha)$

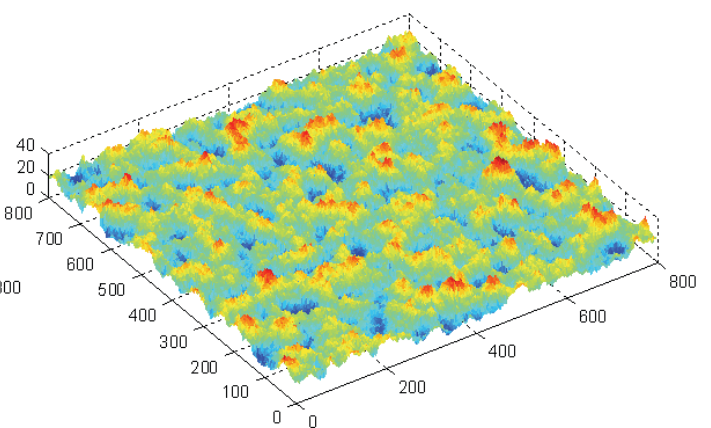

( $\beta)$

Figure 6. Two examples of surfaces generated by the model-aided hybrid method with input the height distribution function and the autocorrelation function shown in Fig. 5.

\section{Summary}

In this work, we propose a model-aided hybrid method for roughness characterization of nanostructured free surfaces. In the proposed method, data from AFM and SEM measurements are synthesized in a synergetic manner to get the increased accuracy merits of AFM height measurements and of SEM spatial arrangement of surface features. The composition is done with the help of a modeling approach enabling the generation of surfaces with input the height distribution function of AFM topographies and the autocorrelation function calculated from top-down SEM images. The generated surfaces can be used to measure more accurately various roughness parameters and metrics and mainly the so-called hybrid parameters which depend on both vertical and spatial aspects of a rough surface.

The critical effect of the application of the proposed hybrid approach to the estimation of the ratio of the active to the nominal area of a film surface is demonstrated for a specific polymer film treated and roughened by oxygen plasma.

The next steps of the further development of the method include: a) More applications and estimation of the uncertainties with respect to single metrologies, b) Benchmarking of the method and comparison with reference standards, c) Effects of AFM tip size on HDF and of 
the SEM image settings on ACF and assessment of their impact on measurement accuracies along with elaboration of remedy strategies.

Finally, it is worth clarifying that the proposed method is not limited to the nanoscale and specific microscopy techniques. It could, for example, be suitably applied to the combination of measuement data from a profilometer and images from an optical microscopy given that the first can provide accurate vertical measurements and the second reliable spatial arrangement of surface morphology.

\section{References}

1. Quere D. 2008 Wetting and Roughness Annu. Rev. Mater. Res. 38 71-99

2. Gogolides E. et al. 2015 Hierarchical micro and nano structured, hydrophilic, superhydrophobic and superoleophobic surfaces incorporated in microfluidics, microarrays and lab on chip microsystems Microelectr. Eng. 132 135-155

3. Ponche A. et al. 2010 Relative influence of surface topography and surface chemistry on cell response to bone implant materials. Part 1: Physico-chemical effects Proc. IMechE Part H: J. Engineering in Medicine 224 1471-1486

4. Chattopadhyay S. et al. 2010 Anti-reflecting and photonic nanostructures Mater. Sci. and Eng. R 69 1-35

5. Yacoot A. and Koenders L. 2008 Aspects of scanning force microscope probes and their effects on dimensional measurement J. Phys. D: Appl. Phys. 41103001 (46pp)

6. Westra K. L. et al. 1993 Tip artefacts in atomic force microscope imaging of thin film surfaces J. Appl.Phys. 74 3608-10

7. Grutter P. et al. 1992 Tip artefacts of microfabricated force sensors for atomic force microscopy Appl. Phys. Lett. 60 2741-3

8. Villarrubia J. S. 1997 Algorithms for scanned probe microscope image simulation, surface reconstruction and tip estimation J. Res. Natl Inst Stand. Technol. 102 245-54

9. Villarrubia J. S. 1996 Scanned probe tip characterization without calibrated tip characterizers J. Vac. Sci. Technol. B 12 1518-21

10. Tranchida D et al. 2006 Some experimental issues of AFM tip blind estimation: the effects of noise and resolution Meas. Sci. Technol. 17 2630-6

11. Marinello F. L. et al. 2008 Critical factors in SEM 3D stereo microscopy Meas. Sci. Technol. 19065705

12. Seeger A. and Haussecker H., 2005 Shape-from-shading and simulation of SEM images using surface slope and curvature Surf. Interface Anal. 37 927-938

13. Foucher J. 2011 Hybrid CD Metrology Concept compatible with high volume manufacturing Metrology, Inspection, and Process Control for Microlithography $X X V$, Proc. SPIE 7971

14. Vaid, A. et al. 2011 A holistic metrology approach: hybrid metrology utilizing scatterometry, CD-AFM, and CDSEM Metrology, Inspection, and Process Control for Microlithography XXV, Proc. SPIE 7971

15. Vaid, A. et al. 2014 Hybrid metrology: from the lab into the fab J. Micro/Nanolith. MEMS MOEMS. 13041410

16. Rana N. et al. 2014 Leveraging advanced data analytics, machine learning, and metrology models to enable critical dimension metrology solutions for advanced integrated circuit nodes J. Micro/Nanolith. MEMS MOEMS. 13041415

17. Hu Y.Z. and Tonder K. 1992 Simulation of 3-D Random Rough Surface By 2-D Digital Filter and Fourier Analysis Int. J. Mach. Tools Manufact. 32 83-90

18. Mack C.A. 2013 Generating random rough edges, surfaces, and volumes Applied Optics 52 1472-1481 